\title{
Understanding Cow Evaluations in Univariate and Multivariate Animal and Random Regression Models
}

\author{
R. Mrode ${ }^{1}$ and M. Coffey \\ Scottish Agricultural College, Sir Stephen Watson Building, Penicuik, EH26 OPH, United Kingdom
}

\begin{abstract}
The relationship between cow evaluations from a 305d lactation yield animal model [i.e., lactation model $(\mathrm{LM})]$ and a random regression model (RRM) were studied using the first-lactation milk yield of $2,477,807 \mathrm{Hol}-$ stein heifers. In the LM analysis, 2 values of heritability were used, $0.35(\mathrm{LM} 1-\mathrm{H})$ or 0.57 (LM2-H), the latter being equal to that used in the random regression model for the analysis of the Holstein test-day records (RRM$\mathrm{H})$. The relative weights on parent average (PA) and yield deviations (YD) were computed and studied to understand factors contributing to reranking of cows' predicted transmitting abilities (PTA) from the various models. The degree of relatedness and inbreeding were calculated for the top 2,000 cows from the various models. Analyses of Jersey milk yield in the first 3 parities was implemented using 305-d lactation yield multivariate animal (MLM-J) and random regression models (MRRM-J). The ability of both models using only firstparity yield records to predict evaluations in second and third parities when records for these later parities were excluded was studied in a sample of cows. The correlations of cow PTA between LM1-H or LM2-H and RRM-H were 0.91 and 0.92 , respectively, in the Holstein data. The data sets used were identical in this case for all models in terms of number of cows and yield records. The correlations were slightly lower at $0.89,0.87$, and 0.88 for parities 1,2 , and 3 in the Jersey analyses, where the data sets were not identical. The relative weights on PA and YD were $0.28(0.11)$ and $0.72(0.89)$, respectively, from the LM2-H (RRM-H). The RRM-H placed more emphasis on YD and therefore on Mendelian sampling deviations. Thus, the top 2,000 cows from the RRM-H were less related and inbred. The average additive genetic relationship was $22 \%$ greater in the LM2-H and average inbreeding coefficients were 0.68 and $0.43 \%$ for the LM2-H and RRM-H, respectively. When records were initially available in the first parity,
\end{abstract}

Received July 6, 2007.

Accepted October 23, 2007.

${ }^{1}$ Corresponding author: Raphael.Mrode@sac.ac.uk the MRRM-J predicted PTA in parities 2 and 3 with about 2 to $7 \%$ greater accuracy compared with the MLM-J.

Key words: random regression, animal model, inbreeding, yield deviation

\section{INTRODUCTION}

One of the criteria examined by countries updating their genetic evaluations from a 305-d lactation yield animal model $(\mathbf{L M})$ to a random regression model (RRM) is the correlations between evaluations from both models. The correlations tend to be high for bulls and usually vary from 0.90 to 0.98 . However, the correlations between cow evaluations are usually lower varying from 0.80 to 0.88 (Reents et al., 1998; Van Doormaal and Kistemaker, 1999; Lidauer et al., 2000), which is indicative of high reranking that may manifest itself especially in top cow lists. This could have implications in terms of selection of bull dams and dams to breed cows and therefore, the efficiency of national breeding programs. Understanding of the factors contributing to this high degree of reranking and its implications are therefore important. Intuitively, a possible explanation is that cow evaluations are based on more limited information relative to bull evaluations and are, therefore, affected more by differences in the models in terms of effects fitted and genetic parameters. However, in a preliminary study, Mrode et al. (2000) suggested that, compared with LM evaluations, RRM places greater emphasis on animal performance relative to pedigree information. The greater emphasis on yield may imply that top lists of cows could include individuals with greater Mendelian sampling effects, cows that are likely to be less related and thus have lower levels of inbreeding.

Second, many countries implement multilactation RRM, and the single published cow evaluations are breeding values averaged across several parities. In the case of young cows, yield information will initially be available in the first parity; therefore, cow evaluations could change over time as additional yield information becomes available in later lactations. Differences in the predictive ability of evaluations in later parities of the 
LM compared with the RRM when yield data are only initially available in the first parity could be important in terms of the overall ranking of cow evaluations from both models.

This study was carried out to examine the relationship between cow evaluations in the top cow lists from the analysis of first-lactation Holstein milk yield fitting an LM with 2 levels of heritability and a RRM. Relative weights on pedigree, yield information, and Mendelian sampling effects were examined to better understand cow evaluations from these models. In addition, relative weights on parent and yield information in a multitrait situation using LM or RRM with milk yield in the first 3 lactations treated as different traits were computed. The predictive abilities of LM and RRM using the first 3 lactations of the Jersey breed when yield information was available only in first parity or in the first 2 lactations were examined. The degree of relatedness and levels of inbreeding were studied in the top 2,000 cows using results from the LM and RRM of the first-lactation analyses of the Holstein.

\section{MATERIALS AND METHODS}

\section{Univariate Analysis of First-Lactation Holstein Data}

The 305-d first-parity milk yield of 2,477,807 Holstein cows from 21,157 herds was analyzed with an LM equivalent to that used in the United Kingdom before the adoption of the RRM (Interbull, 2000). The fixed effects consisted of herd-year-season, age at calving by season of calving, heterosis, and recombination loss. There were 285,488 herd-year-season subclasses present in the data set and a heritability of 0.35 (used in the national LM evaluation) was used (LM1-H). The analysis was repeated using a heritability of $0.57(\mathbf{L M 2}-\mathbf{H})$ computed for 305-d yield from the RRM genetic parameters. The random regression model (RRM-H) used to analyze $23,615,532$ test-day records (TD) for the same set of cows with 1,914,963 herd-test-date subclasses represented, was:

$$
\begin{gathered}
y_{i j k l m t}=h t d_{i}\left(D I M_{t}\right)+h d_{j}\left(D I M_{t}\right)+F i x_{k t} \\
+\sum_{l=0}^{4} \phi_{l m t} \boldsymbol{\beta}_{l}+\sum_{l=0}^{2} \phi_{l m t} \mathbf{u}_{l m} \sum_{l=0}^{2} \phi_{l m t} \mathbf{p}_{l m}+e_{i j k l m t}
\end{gathered}
$$

where $y_{i j k l m t}$ is the TD of cow $m$ made on day $t$ within herd-test-date (htd) subclass $i$ in the $j$ th herd $(\boldsymbol{h d})$; htd (random) and $h d$ (fixed) effects depended linearly on DIM, with an intercept and a slope; Fix $x_{k t}$ are other fixed effects in the model consisting of heterosis, recombination loss calculated from percentage North American Holstein genes and days pregnant by stage of lactation; $\boldsymbol{\beta}_{l}$ are fixed regression coefficients nested within age- season subclasses; $\mathbf{u}_{l m}$ and $\mathbf{p}_{l m}$ are vectors of the $l$ th random regression for animal and permanent environmental effects respectively for animal $m$; $\phi_{l m t}$ is the vector of the $l$ th Legendre polynomial for the test-day record of cow $m$ made on day $t$, and $e_{i j k l m t}$ is the random residual. It is assumed that $\operatorname{var}(\mathbf{u})=\mathbf{A} \otimes \mathbf{G}, \operatorname{var}(\mathbf{p})=$ $\mathbf{I} \otimes \mathbf{P}$ and $\operatorname{var}(\mathbf{e})=\mathbf{R}$ and $\operatorname{cov}(\mathbf{u}, \mathbf{p})=\operatorname{cov}(\mathbf{u}, \mathbf{e})=\operatorname{cov}(\mathbf{p}, \mathbf{e})=$ 0 , where $\mathbf{A}$ is the relationship matrix, $\mathbf{G}$ and $\mathbf{P}$ are the variance and covariance matrices for the random animal and permanent environmental regression effects, respectively, and $\mathbf{R}$ is the diagonal matrix of residual effects. There were 4 classes of residual variances fitted in the RRM-H and these were: $7.86 \mathrm{~kg}^{2}, 4.38 \mathrm{~kg}^{2}$, $3.28 \mathrm{~kg}^{2}$, and $2.89 \mathrm{~kg}^{2}$, for d 4 to 24,25 to 49,50 to 249 , and 250 to 305 in milk, respectively.

All data sets were preadjusted for heterogeneity of variance using the method described by Reents et al. (1998) before analyses using the above models. For the RRM-H, this briefly involved correcting all TD for the stage of lactation, age, and season effects using fixed lactation curves estimated from a fixed effect model. A weighted standard deviation was then calculated for each htd based on an observed standard deviation, expected standard deviation according to the production level to which the htd was assigned, and the herd size. The weighted standard deviation was then used to calculate the standardization factor for each htd. The same procedure was used in the LM except that 305-d milk yield was corrected for age and season effects and herdyear-season subclasses were used instead of htd.

On convergence, 305-d PTA for RRM-H were computed from the regression coefficients for each animal. The PTA were computed from the breeding values from LM1-H and LM2-H. Correlations between cow evaluations from the 3 analyses were computed using all cows with yield information. All PTA were adjusted to the mean of cows born in the base year of 2000 .

\section{Multivariate Analyses of Jersey First Three Lactation Milk Yield}

The first 3 lactation TD milk yields were extracted from the data used for the official genetic evaluation of the Jersey breed in May 2007, and were analyzed using a multivariate random regression model (MRRM-J), regarding milk yield in each lactation as a different trait. The data consisted of 155,137 cows born between 1975 and 2005 with 3,282,323 test-day milk records in the first 3 lactations. The test-day records were from 2,474 herds with 262,585 herd-test-date subclasses represented. The mean number of records per herd-testdate subclass was 22 , with a minimum of 2 and a maximum of 305. The distribution of records per lactation 
Table 1. Summary of test day and $305 \mathrm{~d}$ yields for the Jersey breed in the first three lactations

\begin{tabular}{|c|c|c|c|}
\hline \multirow{2}{*}{$\begin{array}{l}\text { Lactation } \\
\text { number }\end{array}$} & \multicolumn{2}{|c|}{ Test-day yields } & \multirow{2}{*}{$\frac{305-\mathrm{d} \text { yields }}{\text { Cows, } \mathrm{n}}$} \\
\hline & Cows, $\mathrm{n}$ & Test days, $\mathrm{n}$ & \\
\hline 1 & 155,137 & $1,326,371$ & 129,538 \\
\hline 2 & 116,640 & $1,085,483$ & 90,185 \\
\hline 3 & 93,825 & 870,469 & 66,130 \\
\hline
\end{tabular}

is given in Table 1 . The model used was a multivariate extension of model described in equation [1] and it was:

$$
\begin{gathered}
y_{i j k l m q t}=h t d_{i}\left(D I M_{t}\right)+h d_{j}\left(D I M_{t}\right)+F i x_{k q t} \\
+\sum_{l=0}^{4} \phi_{l m q t} \boldsymbol{\beta}_{l q}+\sum_{l=0}^{2} \phi_{l m q t} \mathbf{u}_{l m q}+\sum_{l=0}^{2} \phi_{l m q t} \mathbf{p}_{l m q}+e_{i j k l m q t}
\end{gathered}
$$

where $y_{i j k l m q t}$ is the TD of cow $m$ for trait $q$ made on day $t$ within $h t d$ subclass $i$ in the $j$ th herd. The $h t d$ and $h d$ effects were as defined in [1] and were fitted across the 3 traits, but Fix $x_{k q t}$ is now the fixed effect of days pregnant by stage of lactation for the $q$ th trait. All other effects in [2] for the $q$ th trait were as defined in model [1]. The variance and covariance structure assumed was as defined in [1] but were now for 3 traits. There were 4 classes of residual variances fitted for each parity as described for model [1]. After convergence, PTA for 305-d yield in each lactation were computed from the regression coefficients for each animal.

The data were reanalyzed with test-day records omitted in later parities for all cows born after December 31,2000 . In the first analysis, TD records were omitted from both the second and third parities for these cows (MRRM1-J); then, in a second analysis, TD records were only omitted from the third parity (MRRM2-J). The ability of the MRRM-J to predict PTA in the second and third parities when TD records were excluded was studied in a random sample of 1,300 cows that had their second and third or only third lactation TD records deleted. For this sample of cows, their progeny were also excluded from the data. This was to ensure that PTA values predicted for the later parities of these cows when observations were excluded did not contain contributions from progeny. The PTA from MRRM1-J and MRRM2-J for this sample of cows were correlated with and regressed on PTA from MRRM-J after all PTA had been adjusted to the same base.

Corresponding multivariate animal model evaluations were implemented on the same data using 129,538 cows that had completed 305-d yield records. The 305$\mathrm{d}$ records were from 1,738 herds with 38,040 herd-yearseason subclasses represented. The mean number of records per hear-year-season subclass was 7 , with a minimum of 2 and a maximum of 165 . The multivariate
Table 2. Genetic parameters for 305-d milk yield in the first 3 lactations showing heritabilities along the diagonal (in boldface) and genetic and phenotypic correlations below and above the diagonal, respectively

\begin{tabular}{lccc}
\hline Parity & 1 & 2 & 3 \\
\hline 1 & $\mathbf{0 . 5 7}$ & 0.54 & 0.49 \\
2 & 0.76 & $\mathbf{0 . 5 6}$ & 0.63 \\
3 & 0.69 & 0.95 & $\mathbf{0 . 6 0}$ \\
\hline
\end{tabular}

lactation animal model (MLM-J) used for the analyses consisted of herd-year season, month of calving, and age effects as fixed effects, and random effects were animal and permanent environmental effects. The permanent environmental effects were equivalent to those fitted for the MRRM-J and this implies that residual variances in all 3 parities were uncorrelated. The genetic parameters used for the analyses were computed from the random regression parameters (Thompson et al., 2005) and are shown in Table 2. Multivariate lactation animal models with $305-d$ yields omitted in parities 2 and 3 (MLM1-J) or in only parity 3 (MLM2-J) for cows born after December 31, 2000, were also implemented. Again, the sample of 1,300 cows that had both their second and third or only third lactations deleted and with their progeny excluded from the data sets was also studied. The PTA in the 3 parities from MLM-J were averaged and correlated with equivalent ones from MRMM-J. The PTA from MLM1-J and MLM2-J were correlated with, and regressed on, those from MLM-J in the sample of 1,300 cows. A summary of the various data analyses implemented, models and abbreviations are shown in Table 3.

\section{Computing Relative Weights for Parent Average and Yield Deviation}

For a cow with no progeny, the equation of VanRaden and Wiggans (1991) was used to compute the relative weight on pedigree and yield information in LM1-H and LM2-H:

$$
\mathrm{PTA}_{\text {cow }}=\mathrm{W}_{1}(\mathrm{PA})+\mathrm{W}_{2}(\mathrm{YD} / 2)
$$

where PA is the parent average PTA, YD is the yield deviation that represents the yield of the animal adjusted for all effects in the model other than the genetic merit and error, and $\mathrm{W}_{1}$ and $\mathrm{W}_{2}$ are relative weights for PA and YD, respectively, that sum to unity. The numerators of $\mathrm{W}_{1}$ and $\mathrm{W}_{2}$ are $\alpha u_{\mathrm{par}}$ and $n$ respectively, with $\alpha=\sigma_{e}^{2} / \sigma_{a}^{2}, u_{\mathrm{par}}=2,4 / 3$, or 1 when both, 1 , or neither parents are known, and $n$ is the number of records. The denominator of either $\mathrm{W}_{1}$ or $\mathrm{W}_{2}$ is the sum of the 2 numerators. The relative weight for Mendelian sam- 
Table 3. Summary of data, models and abbreviations for various analyses implemented

\begin{tabular}{ll}
\hline Abbreviation & Analyses \\
\hline $\begin{array}{l}\text { Analyses on Holstein data } \\
\text { LM1-H }\end{array}$ & Animal model for Holstein first-lactation milk yield, heritability of 0.35 \\
LM2-H & Animal model for Holstein first-lactation milk yield, heritability of 0.57 \\
RRM-H & Random regression model for Holstein first-lactation milk yield \\
Analyses on Jersey data set & Multivariate animal model for Jersey first 3 lactation milk yield \\
MLM-J & Multivariate animal model for Jersey first 3 lactation milk yield with records for second and third \\
MLM1-J & parities deleted for cows born after the year 2000 \\
MLM2-J & Multivariate animal model for Jersey first 3 lactation milk yield with records for third parities \\
deleted for cows born after the year 2000 & Multivariate random regression model for Jersey first 3 lactation milk yield \\
MRRM-J & Multivariate random regression model for Jersey first 3 lactation milk yield with records for second \\
MRRM1-J & and third parities deleted for cows born after the year 2000 \\
MRRM2-J & Multivariate random regression model for Jersey first 3 lactation milk yield with records for third \\
& parities deleted for cows born after the year 2000 \\
\hline
\end{tabular}

pling (MS) was calculated by expressing [3] (Mrode, 2005) as:

$$
\mathrm{PTA}_{\text {cow }}=\mathrm{PA}+\mathrm{W}_{2}(\mathrm{MS})
$$

Equivalent equations to [3] and [4] (Mrode and Swanson, 2004) were used to calculate relative weights for PA and YD or MS for the RRM-H. Similar to the definition in [3], YD in the RRM-H case is a vector of the weighted average of a cow's TD corrected for all fixed and permanent environmental effects. The weighting is based on the inverse of the residual variance relevant to the TD in the different stages of the lactation. However, these relative weights are matrices of order 3 . The use of Legendre polynomials in [1] implies that the majority of the information for 305-d evaluations computed from the random regression coefficients for animals would be coming from the relative weights on the intercept for PA and YD. Thus, the relative weights on the intercept were used as approximate weights on PA and YD. This is equivalent to solving the multivariate equations equivalent to [3] (Mrode and Swanson, 2004) by assigning the same value of $\left[\begin{array}{lll}1 & 0 & 0\end{array}\right]$ to $\mathbf{P A}^{\prime}$ and $\mathbf{Y D}^{\prime}$. The summation of the actual information contributed by PA and YD as a ratio of overall PTA over a large number of cows should approximate $\mathrm{W}_{1}$ and $\mathrm{W}_{2}$ calculated using equations [3] and [4]. This was done for LM1-H, LM2-H, and RRM-H, and the summation was over 1,434,516 Holstein cows with no progeny.

On the basis of the models used for the multivariate analyses of Jersey milk yield in the first 3 lactations as different traits, equivalent equations to [3] (Mrode and Swanson, 2004) were set up to compute relative weights on PA and YD for MLM-J and MRRM-J. From [2], Legendre polynomials of order 2 were used for each lactation; therefore, the genetic covariance for random regression coefficients for animal effects was of order 9 for the 3 lactations. Similarly, the weights on PA $\left(\mathrm{W}_{1}\right)$ and YD $\left(\mathrm{W}_{2}\right)$ for the MRRM-J were matrices of order 9 but for MLM-J, these matrices were of order 3. In the case of MLM-J, the relative weights for PA and YD in each lactation were obtained by solving the multivariate equations equivalent to [3] by assigning the same values to PA and YD. In the case of the MRRM-J, the approximate relative weights based mainly on the intercept were computed by assigning the same value of $\left[\begin{array}{llllllll}1 & 0 & 0 & 1 & 0 & 0 & 1 & 0\end{array}\right]$ to $\mathbf{P A}^{\prime}$ and $\mathbf{Y D}^{\prime}$.

\section{Relative Contribution of PA and YD}

Using [3] and [4], the relative contributions from PA, $\mathrm{YD}$, and MS were computed and studied in a random sample of 7,500 Holstein cows in the evaluations from LM2-H and RRM-H. The influence of these relative contributions on the reranking of cow evaluations was examined in this sample of cows.

\section{Degree of Relatedness}

A 4-generation pedigree was extracted for the top 2,000 cows in each of the 3 models (RRM-H, LM1-H, and LM2-H) used to analyze the first-lactation yield of the Holstein data, and the degree of relatedness and inbreeding were computed for the cows in these datasets.

\section{RESULTS AND DISCUSSION}

\section{Correlations Between Cow Evaluations}

The correlation between evaluations for Holstein cows from LM1-H and LM2-H with RRM-H were 0.91 and 0.92 , respectively. These are slightly greater than the estimates of Reents et al. (1998), Van Doormaal and Kistemaker (1999), and Lidauer et al. (2000). This could be due to the fact that both models were applied to identical data sets. This would not be the case in 
Table 4. The relative weights on parents' average $\left(\mathrm{PA}^{1}\right)$ in different lactations from multitrait equations for 305-d lactation-yield animal model (MLM-J) or random regression model (MRRM-J)

\begin{tabular}{|c|c|c|c|c|c|c|c|}
\hline \multirow{2}{*}{$\begin{array}{l}\text { Parity } \\
\text { no. }\end{array}$} & \multirow{2}{*}{$\begin{array}{l}\text { Test } \\
\text { days, }{ }^{2} \mathrm{n}\end{array}$} & \multicolumn{3}{|c|}{ MRRM-J } & \multicolumn{3}{|c|}{ MLM-J } \\
\hline & & $\mathrm{PA}_{1}$ & $\mathrm{PA}_{2}$ & $\mathrm{PA}_{3}$ & $\mathrm{PA}_{1}$ & $\mathrm{PA}_{2}$ & $\mathrm{PA}_{3}$ \\
\hline 1 & 3 & 0.39 & 0.49 & 0.45 & & & \\
\hline 1 & 6 & 0.22 & 0.31 & 0.26 & & & \\
\hline 1 & 8 & 0.16 & 0.24 & 0.20 & & & \\
\hline 1 & 10 & 0.11 & 0.19 & 0.15 & 0.28 & 0.33 & 0.29 \\
\hline 2 & 10 & 0.09 & 0.03 & 0.03 & 0.20 & 0.06 & 0.06 \\
\hline 3 & 10 & 0.09 & 0.04 & 0.01 & 0.20 & 0.06 & 0.01 \\
\hline
\end{tabular}

national evaluation schemes, because the RRM may include cows with TD records from uncompleted lactations, which would not be in the LM analysis. On the other hand, cows with only 305-d yield records and no TD records would only be included in the LM evaluations and not in the RRM evaluations. Thus, parent and progeny contributions to the evaluations of the same cows in both models will be different for some animals. The correlations of Jersey cow PTA from the multivariate analysis, MLM-J, which used data in all lactations with those from the MRRM-J, were 0.89, 0.87 , and 0.88 , respectively, for the first, second, and third parities. The correlation was 0.88 for a PTA averaged across the 3 parities. These are more similar to estimates reported in the literature because data sets were not identical for both models. The MRRM-J included TD records from part-lactation yields, whereas these cows were not in the data set for MLM-J. These correlations confirm substantial reranking among cows across all 3 parities between both models.

\section{Computing Relative Weights for PA and YD}

The relative weights from the univariate analysis on PA and YD from [3] were 0.74 and 0.26 for LM1-H. Corresponding estimates were 0.28 and 0.72 for LM2$\mathrm{H}$. The information contributed by $\mathrm{PA}$ or $\mathrm{YD}$ as a ratio of overall PTA summed over the 1.4 million cows was 0.68 or 0.22 , respectively, for LM1-H. Similar estimates for PA and YD were 0.31 and 0.69 for LM2-H, and these are not very different from the relative weights calculated using equation [3]. For the RRM-H, the relative weights for a cow with 10 test-day records approximated by the weights on the intercept were 0.11 for PA and 0.89 for YD. The ratio of the actual contribution from PA or YD to 305-d PTA summed over the 1.4 million cows was 0.14 or 0.86 , respectively. These are not significantly different from the relative weights based on the intercept. The relative weights on PA and YD obtained using [3] for LM1-H, LM2-H, and equivalent equations for RRM-H were based on a situation in which no inbreeding was assumed in calculating $\mathbf{A}^{-1}$. If inbreeding was accounted in $\mathbf{A}^{-1}$ and an inbreeding coefficient of 0.125 , for instance, was assumed for an animal, the relative weights obtained using [3] were similar to the situation with no inbreeding. The relative weights on PA increased to 0.75 for LM1-H and 0.30 for LM2-H, but remained unchanged at 0.11 for RRM-H.

Thus, as expected, as heritability increased (LM1-H vs. LM2-H), the relative weight on pedigree information decreased in the LM analyses. However, a greater reduction on the relative weight on PA with the RRM-H, indicates that at the same level of heritability, RRM$\mathrm{H}$ placed more emphasis on YD and, therefore, on MS. In fact, the relative weight on PA for a cow with $4 \mathrm{TD}$ records in the RRM-H is equivalent to that in LM2-H in which a whole lactation has been used. Thus, at the same level of heritability, the emphasis placed on pedigree and yield in the LM2-H was similar to that in the RRM-H for a cow with just 4 TD records. The use of individual TD records in the RRM-H represents better use of information compared with one record in the LM2-H, thus evaluations are less regressed to the parent average in the RRM-H (Lidauer, 2002). This explains why the standard deviations of evaluations for the RRM are usually larger than those from the LM, even at the same level of heritability (Lidauer, 2002). The standard deviation of cow evaluations in the RRM$\mathrm{H}$ in this study was about $20 \%$ greater than in the LM2$\mathrm{H}$. This is comparable to the increase of about 15 to $20 \%$ reported by Lidauer (2002).

The relative weights on PA and yield information in the first 3 lactations from the multivariate analyses on the Jersey data set are shown in Table 4. Also shown in Table 4 is the relative weight on PA in the first 3 lactations as the number of test-day records increases in the first parity. As more TD records become available in the first lactation, the relative emphasis on PA decreases in all 3 lactations. This is even more so as yield data become available in second and third parities. However, emphasis on PA is still greater by about twice in the MLM-J compared with the MRRM-J. In both 
Table 5. Yield deviations, PTA, and proportion contributed by Mendelian sampling (MS) for 3 categories of cows ${ }^{1}$

\begin{tabular}{|c|c|c|c|c|c|c|}
\hline \multirow[b]{2}{*}{ Cows } & \multicolumn{2}{|c|}{ Yield deviation (kg) } & \multicolumn{2}{|c|}{ PTA (kg) } & \multicolumn{2}{|c|}{ MS } \\
\hline & LM2-H & RRM-H & LM2-H & RRM-H & LM2-H & RRM-H \\
\hline \multicolumn{7}{|c|}{ Category A ${ }^{2}$} \\
\hline Cow 1 & 945 & 923 & 113.3 & 189.8 & 0.18 & 0.41 \\
\hline Cow 2 & 961 & 981 & 178.2 & 350.3 & 0.05 & 0.25 \\
\hline \multicolumn{7}{|c|}{ Category $\mathrm{B}^{3}$} \\
\hline Cow 1 & 1,011 & 1,019 & 207.0 & 153.3 & 0.10 & 0.09 \\
\hline Cow 2 & 228 & 232 & -508.9 & -601.0 & -0.28 & -0.58 \\
\hline \multicolumn{7}{|c|}{ Category $\mathrm{C}^{4}$} \\
\hline Cow 1 & 898 & 904 & 65.4 & 61.8 & 0.21 & 0.24 \\
\hline Cow 2 & 1,199 & 1,227 & 369.3 & 367.0 & 0.14 & 0.16 \\
\hline
\end{tabular}

${ }^{1}$ See Table 3 for definitions of abbreviations.

${ }^{2} \mathrm{PTA}$ values greater in RRM1 than in LM2-H.

${ }^{3} \mathrm{PTA}$ values greater in LM2-H than in RRM-H.

${ }^{4} \mathrm{PTA}$ values very similar in RRM-H and LM2-H.

models, relative weight on PA is lower in parities 2 and 3 compared with first parity. This could be due to the greater heritability in the third parity and the high correlation of 0.95 between second and third parity in this study.

\section{Relative Contribution of PA and YD}

For the sample of 7,500 Holstein cows studied, differences in YD were the main factor influencing the relative ranking of cows in the LM2-H and RRM-H. For instance, cows with greater 305-d YD in the RRM-H tended generally to have greater PTA predicted in the RRM-H relative to LM2-H and vice versa. However, for cows with similar YD on a $305-\mathrm{d}$ basis ( $\leq 5 \%$ different) in the RRM-H and LM2-H, the role of the relative contribution of PA and MS on PTA is shown in some chosen cows (Table 5) that best illustrate the principle. Cows with greater PTA in the RRM-H ( $>50 \mathrm{~kg}$; category A) had greater (about 30 to $80 \%$ greater) positive MS relative contribution in the RRM-H. The relative MS contribution summed over 2,500 sampled cows that fell into this category was 0.14 for LM2-H and 0.22 for RRM$\mathrm{H}$. For cows with lower PTA in the RRM-H ( $\leq 50 \mathrm{~kg}$; category B), this was either because of a similar MS relative contribution in both models, such that a greater emphasis on PA resulted in a greater PTA in the LM2$\mathrm{H}$ or a greater negative MS contribution in the RRM$\mathrm{H}$. The relative MS contribution summed over 2,500 of the sampled cows that fell into this category was -0.04 for LM2-H and -0.13 for RRM. Category $\mathrm{C}$ cows had similar PTA in both models ( $\pm 10 \mathrm{~kg}$ ), and the MS contribution was also very similar. The relative MS contribution summed over 2,500 of the sampled cows in this category was 0.21 for LM2-H and 0.20 for RRM-H.

\section{Predictive Ability}

Table 6 shows the mean differences between PTA by parity (MLM1-J - MLM-J and MRRM1-J - MRRM-J) for the sample of 1,300 cows when records were excluded for parities 2 and 3. Also shown in Table 6 are the correlations between PTA from MLM-J and MLM1J, and MRRM-J and MRRM1-J by parity. The mean differences indicate a slight underprediction in using only first-parity yield data to predict PTA in later lactations. The underprediction varied from 6 to $19 \mathrm{~kg}$ of milk for the second parity and about $25 \mathrm{~kg}$ for the third parity. However, given the standard deviations of the differences, these mean differences were not significant.

Table 6. Mean differences, correlations, and regressions of PTA from evaluations with yield data omitted from parities 2 and 3 with those from evaluations with no loss of data in a sample of 1,300 cows $^{1}$

\begin{tabular}{lccccccc}
\hline & \multicolumn{3}{c}{ MLM1-J } & & \multicolumn{3}{c}{ MRRM1-J } \\
\cline { 2 - 4 } \cline { 7 - 8 } Item & Parity 1 & Parity 2 & Parity 3 & & Parity 1 & Parity 2 & Parity 3 \\
\hline Mean difference & $-0.55(56)^{2}$ & $-6.40(176)$ & $-25.44(222)$ & & $-4.79(62)$ & $-19.68(151)$ & $-26.88(177)$ \\
Correlation & 0.98 & 0.84 & 0.78 & & 0.97 & 0.86 & 0.85 \\
Regression & 1.00 & 0.96 & 0.93 & & 1.00 & 1.00 & 1.00 \\
\hline
\end{tabular}

${ }^{1}$ See Table 3 for definitions of MLM1-J and MRRM1-J.

${ }^{2}$ Standard deviations in parentheses. 
Table 7. Mean differences (kg), correlations, and regressions of PTA from evaluations with yield data omitted from parity 3 only with those from evaluations with all data in a sample of 1,300 cows

\begin{tabular}{lccclccc}
\hline & \multicolumn{3}{c}{ MLM2-J } & & \multicolumn{3}{c}{ MRRM2-J } \\
\cline { 2 - 4 } Item & Parity 1 & Parity 2 & Parity 3 & & Parity 1 & Parity 2 & Parity 3 \\
\hline Mean difference & $-0.32(35)$ & $-0.31(99)$ & $-17.27(155)$ & & $2.37(63)$ & $-7.29(91)$ & $-12.94(115)$ \\
Correlation & 0.99 & 0.95 & 0.90 & & 0.99 & 0.97 & 0.95 \\
Regression & 1.00 & 0.99 & 0.98 & & 1.00 & 0.99 & 0.99 \\
\hline
\end{tabular}

${ }^{1}$ See Table 3 for definitions of MLM2-J and MRRM2-J.

${ }^{2}$ Standard deviations in parentheses.

The lower standard deviations of the differences for the MRRM-J are, however, indicative of the superior predictive ability of the random regression model. This result is consistent with the greater correlations between PTA MRRM-J and MRRM1-J in parities 2 and 3 compared with equivalent PTA from the multivariate lactation animal model. The regression of PTA from MRRM-J on MRRM1-J gave unity regression coefficients (Table 6), whereas those from the lactation model varied from 0.93 to 0.96 . When data were omitted only in the third parity (Table 7), a similar trend was observed with predictions in the third parity for the sample of cows for both models. The correlation was greater in the third parity with the MRRM2-J but the regression was very close to unity. In general, it seems that the MRRM-J model predicted PTA in later parities with an accuracy that was about 2 to $7 \%$ greater than with the MLM-J. Thus, differences in the predictive ability of evaluations in later parities when data are not yet available would be contributory factors to the reranking in cow evaluations from both models.

\section{Degree of Relatedness}

With less emphasis on parent information, we expected the RRM-H to result in the selection of top cows that were less related and therefore less inbred compared with LM1-H and LM2-H. Summary statistics on the level of relatedness and inbreeding in the top 2,000 cows from the 3 univariate analyses on the Holstein data set are given in Table 8 . The RRM-H resulted in

Table 8. Summary of results on the degree of relatedness for the top 2,000 Holstein cows from the lactation models and random regression model $^{1}$

\begin{tabular}{lccc}
\hline Item & LM1-H & LM2-H & RRM-H \\
\hline No. of sires & 249 & 384 & 442 \\
No. of dams & 1,832 & 1,886 & 1,944 \\
No. related & 1,836 & 1,716 & 1,536 \\
Average relationship (\%) & 4.4 & 2.7 & 2.1 \\
No. inbred & 1,005 & 870 & 675 \\
Average inbreeding (\%) & 0.72 & 0.68 & 0.43 \\
\hline
\end{tabular}

${ }^{1}$ See Table 3 for definition of LM1-H, LM2-H, and RRM-H. an increase in the number of sires represented in the top 2,000 cows by 78 and $15 \%$ compared with LM1-H and LM2-H, respectively, thereby increasing the genetic selection base. However, corresponding increases in the number of dams represented were only 6 and $3 \%$ relative to LM1 and LM2-H. The average additive genetic relationships between the cows in LM1-H was twice that in RRM-H but was only $22 \%$ greater in LM2$\mathrm{H}$ than RRM-H. The number of related individuals decreased from 1,836 in LM1-H to 1,716 in LM2-H and 1,536 in the RRM-H with corresponding average inbreeding coefficients of $0.72,0.68$, and $0.43 \%$, respectively. The proportion of the top 2,000 cows with greater-than-zero inbreeding coefficients were 0.50 , 0.44 , and 0.34 for LM1, LM2, and RRM-H, respectively. The distribution of cows by the level of inbreeding coefficient indicated that at each level of inbreeding ranging from 1 to $13 \%$, there were fewer cows from the RRM$\mathrm{H}$ compared with LM1-H and LM2-H. This would imply that progeny bred from the top 2,000 cows, given the same sires, should be less related, have lower inbreeding levels, and therefore less inbreeding depression with the RRM-H compared with LM1-H or LM2-H. However, given the greater emphasis on Mendelian sampling effects by the RRM-H, it is not clear whether estimates of inbreeding depression from including inbreeding in the model as covariate will differ between an LM and RRM, in a given population. This is an area that could be investigated further.

\section{CONCLUSIONS}

Factors contributing to the differences in the ranking of top cows between the LM and RRM include the greater emphasis on yield deviation and Mendelian sampling, even at the same level of heritability. In a multivariate situation when data are only initially available in first parity, differences in the predictive ability of evaluations in later parities may be another factor. The greater emphasis on yield deviation in the RRM resulted in the selection of top cows with greater Mendelian sampling and these were less related. This could result in slightly more genetic progress in the 
dam pathway to breed bulls and cows, but could also be a problem if there is preferential treatment of bull dams. For countries moving from LM to RRM, the difference in heritability estimates used in both models is another contributing factor to differences in cow ranking.

\section{REFERENCES}

Interbull. 2000. National genetic evaluation programmes for dairy production traits practiced in Interbull member countries 1999 2000. Interbull Bull. 24:47-52.

Lidauer, M. 2002. Random regression for the genetic evaluation in dairy cattle. PhD thesis. University of Agricultural Sciences, Vienna, Austria.

Lidauer, M., E. A. Mäntysaari, I. Stradén, and J. Pösö. 2000. Multipletrait random regression test day model for all lactations. Interbull Bull. 25:81-86.
Mrode, R. A. 2005. Linear Models for the Prediction of Animal Breeding Values. 2nd ed. CABI International, Wallingford, UK.

Mrode, R. A., and G. J. T. Swanson. 2004. Calculating cow and daughter yield deviations and partitioning of genetic evaluations under a random regression model. Livest. Prod. Sci. 86:253-260.

Mrode, R. A., G. J. T. Swanson, and C. M. Lindberg. 2000. Relative weights on pedigree information and performance in evaluations based on a test day model. Page 38 in Proc. Br. Soc. Anim. Sci., York, UK

Reents, R., L. Dopp, M. Schmutz, and F. Reinhardt. 1998. Impact on application of a test day model to dairy production traits on genetic evaluations of cows. Interbull Bull. 17:49-54.

Thompson, R., S. Brotherstone, and I. M. S. White. 2005. Estimation of quantitative genetic parameters. Phil. Trans. R. Soc. 360:1469-1477.

Van Doormaal, B. J., and G. J. Kistemaker. 1999. Application of the Canadian test day model. Interbull Bull. 22:100-103.

VanRaden, P. M., and G. R. Wiggans. 1991. Derivation, calculation and use of national animal model information. J. Dairy Sci. 74:2737-2746. 